\title{
A PILOT STUDY ON THE EFFECT OF ORAL DENTIFRICE ON SALIVARY AMYLASE
}

\author{
Sravanti Vaidya ${ }^{1}$, Sweekruthi $A^{2}$, Snehal $V^{3}$, Navitha $I^{4}$, Nethravathy ${ }^{5}$ \\ ${ }^{1}$ Department of Biotechnology, M. S. Ramaiah Institute of Technology, Bangalore-560054 \\ ${ }^{2}$ Department of Biotechnology, M. S. Ramaiah Institute of Technology, Bangalore-560054 \\ ${ }^{3}$ Department of Biotechnology, M. S. Ramaiah Institute of Technology, Bangalore-560054 \\ ${ }^{4}$ Department of Biotechnology, M. S. Ramaiah Institute of Technology, Bangalore-560054 \\ ${ }^{5}$ Department of Biotechnology, M. S. Ramaiah Institute of Technology, Bangalore-560054
}

\begin{abstract}
Saliva is an important body fluid which aids in digestion, in providing a first line of defense and has been recently identified as a repertoire of biomarkers. Salivary composition, hormone levels and enzyme activities are greatly influenced by physiology and well being of the individuals. Oral hygiene is generally maintained by the use of Oral dentifrice like toothpaste and mouthwash. These oral dentifrice which mainly provide antimicrobial property also have other added reagents whose role on saliva is not yet clear. Therefore, the current work deals with the study of, the effect of oral dentifrices on the activity of salivary amylase; one of the most abundant enzymes in the saliva. Several mouthwashes and toothpastes having natural or synthesized active ingredients were used in this work. Saliva samples of individuals were collected before and after use of the oral reagent and amylase activity pre and post rinse was measured by DNS method. Our results have shown that Himalaya, Colgate total, Himalaya sparkling white toothpastes decrease amylase activity where as Pepsodent, Colgate visible white increase salivary amylase activity. In the case of mouthwash use of Listerine, Freshclor, Hiora increases the amylase activity. These results indicate that oral dentifrices influence the salivary amylase activity. This is a pilot study which can be extended to a bigger sample size and can include more dentifrices to provide a comprehensive understanding of the effect of various oral reagents on salivary constituents.
\end{abstract}

Keywords: Oral dentifrice, Saliva, Amylase, DNS

\section{INTRODUCTION}

Saliva is a body fluid which is secreted mainly by the parotid, submaxillary and sublingual glands in humans. The minor salivary glands are Lingual mucus glands, Lingual serous glands, Buccal glands, Labial glands, Palatal glands.(Tina, Justin, et al ,2011). Saliva performs various functions from assisting in digestion to providing a first line of defence to microrganism. Saliva is composed of several proteins, hormones and salts (Zelles,Purushotham,et al,1995). Mixed saliva contains 99.5\% water and $0.5 \%$ solids. DHEA, Estradiol, Cortisol, CRP, Neopterin, IL6, IgA, Cathepsin, Lysozymes are important constituents of saliva. (Chicharro, Lucia, et al, 1998). Generally physiological changes in the body are reflected as a change in the salivary composition. Several saliary biomarkers have been identified which have paved way for development of Salivary diagnostic tools. Salivary diagnostics are now being applied to detect stress, reproductive health disorders, inflammation, child development, behavioural disorders, sleep and circadian rhythm changes, emotions, pregnancy, aging, menopause, Cancer, Inflammation, Obesity and Diabetes, disorders like Cushings Syndrome, Chronic Fatigue Syndrome, Muscular Dystrophy, Seasonal Affective Disorder, Multiple Sceloris, HIV and other systemic disorders (Kaufman,Lamster,2002) (Tina Pfaffe, Justin
Cooper, et al,2011). Salivary diagnostic approaches have been developed to monitor oral diseases such as periodontal diseases and to assess caries risk (Kornman KS, Crane A, Wang HY,1997).

Salivary Amylase is one of the most abundant enzymes in saliva. It is an enzyme which is not only important for oral digestion but also plays a vital role in the formation of dental plaque.

Dental plaque can lead to Gingivitis, bad odour, and tooth decay. It has been shown by earlier work that salivary alpha amylase assists the binding of bacteria to the teeth and inturn aid in dental plaque formation. Hence inhibition of Salivary Amylase is essential for prevention of dental caries and plaque associated oral diseases (Mojarad. et al., 2013).

To prevent formation of plaques and calculus oral hygiene is generally maintained by mechanical brushing and use of chemical oral dentifrice like toothpastes and mouth wash Gunsolley JC. (2006). Oral dentifrice include several antimicrobial, antifungal, antisepctic, anti-inflammatory, abrasive, foaming agents apart from other chemicals whose 
exact role on saliva is not yet studied. The market is currently flooded with several oral dentifrice which claim to improve oral hygiene. Therefore the aim of the current study is to understand the influence of few toothpastes and mouthwashes on the activity of salivary amylase.

\section{MATERIALS AND METHODS}

Various oral reagents considered for the current research worksare Listerine, HiOra mouth wash, Colgate Plax Sensitive and Freshclor. The toothpastes includes Colgate total advanced health, Pepsodent germi check, Himalaya herbal gum expert, Colgate visible white and Himalaya sparkling white.

Salivary samples were collected before and after rinse/brush (15 minutes later) with mouthwash/toothpaste and stored at $4{ }^{\circ} \mathrm{C}$ in clean vials until sample preparation. Saliva sample thus stored were centrifuged at $5000 \mathrm{rpm}$ for 10 minutes and the supernatant was used for further experiments. Saliva samples were diluted by phosphate buffer $\mathrm{pH} 6.5$ tested for Amylase activity. Salivary Amylase activity in saliva was tested in duplicates by standard DNS method.

\section{RESULTS AND DISCUSSION}

A set of standards were measured and a Maltose Standard curve was generated using $0.1 \mathrm{~g}$ of maltose as shown in Fig 1. OD values obtained at $540 \mathrm{~nm}$ by DNS method were plotted across the maltose standard plot to calculate amylase activity.

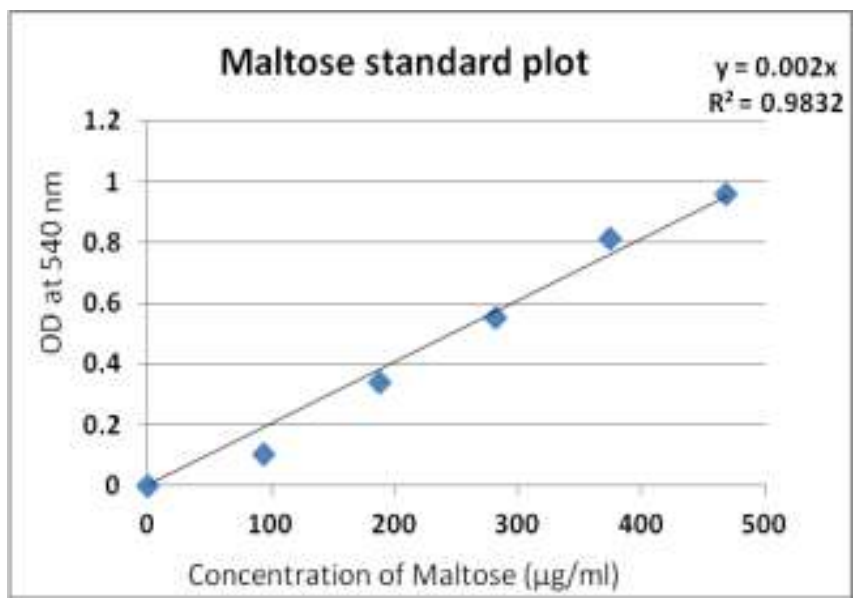

Fig 1: Maltose standard plot

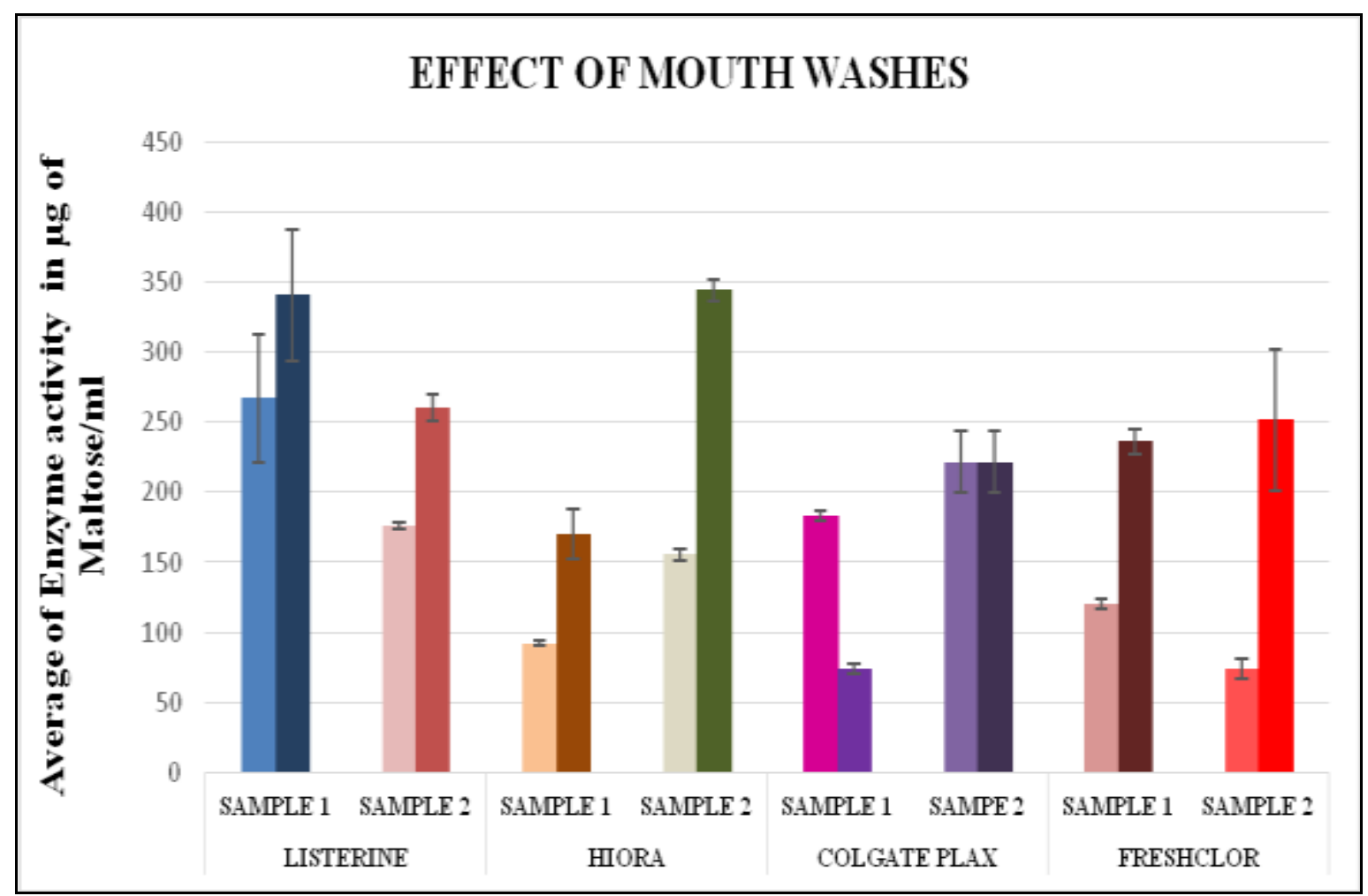




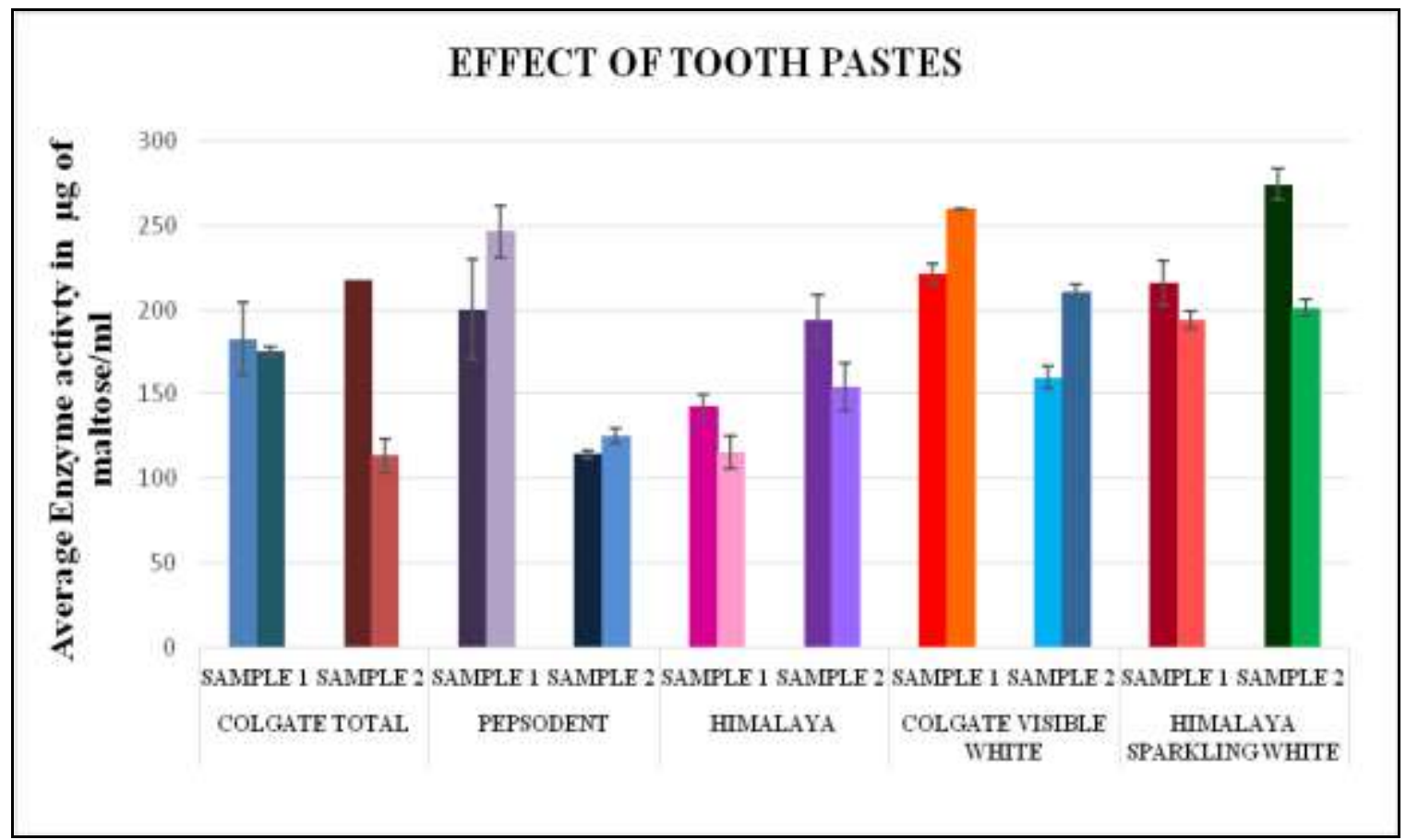

Fig 2: Comparison of effect of various mouthwashes and toothpastes on Amylase. In each sample the left bar indicates pre-rinse/prebrush amylase activity and the right bar indicates post-rinse/post-brush amylase activities.

All experiments were performed in duplicates and standard deviations were calculated. It was interesting to note that salivary amylase activity was different for different samples. Also same individual showed slight variations in enzyme activity on different days. Therefore intrinsic differences in activity of amylase in different individuals reflects on the changes in metabolism

The activity of salivary amylase was tested in the pre and post samples for each of the toothpaste and mouthwash. It was observed that the use of oral dentifrice does influence the activity of amylase in all the cases. Overall use of Listerine, HiOra, Freshchlor mouthwashes and Pepsodent and Colgate visible white toothpastes increased the salivary amylase activity. On the other hand use of Himalaya gum expert toothpaste and Colgate plax mouthwash decreased the amylase activity. This indicates that the reagents used in the development of these dentifrices have an influence on saliva apart from providing antimicrobial properties and assisting in maintaining oral hygiene.

\section{CONCLUSION}

Our results indicate that among toothpastes Pepsodent and Colgate visible white increase the amylase activity while Himalaya gum expert and decrease activity. In the mouthwash Listerine, HiOra, Freshchlor increase amylase activity whereas Colgate Plax decreases amylase activity. Thus oral dentifrices do influence salivary amylase activity.

Further it will be interesting to identify the role of major ingredients of the oral dentifrice and their effect on other Salivary enzymes. All this data will be of great benefit to the dentists and oral hygiene product manufacturing companies which will in turn have an impact on the lives of common people.

\section{REFERENCES}

[1] Chicharro JL, Lucia A, Perez M, Vaquero A F, Urena R.(1998). Saliva Composition And Exercise. Sports Medicine,26,17-27.

[2] Gunsolley JC. (2006). A meta analysis of six month studies of antiplaque formation and antigingivitis agents, Journal of the American Dental Assosiation, 137(123),1649-1657.

[3] Kornman KS, Crane A,Wang HY(1997).The interleukin-1 genotype as a severity factor in adult periodontal disease.Clinical Periodontitis,24,72-77.

[4] Matthews RW.(2003).Hot salt water mouth baths. British Dental Journal,195(1),3.

[5] Mojarad. F, Faziollahifar. S, Poorolajal. J, Hajilooi. M (2013) Effect of alpha amylase on early childhood caries: a matched case-control study. Braz. Dent. Sci 16: $41-45$

[6] Tina Pfaffe, Justin Cooper-White, Peter Beyerlein, Karam Kostner, Chamnindie Punyadeera. (2011), Clinical Chemistry, 57(5),675-687.

[7] Zelles,Purushotham,Macauley .(1995).Saliva and growth factors:the fountain if youth resides in all. Jouranal of dental science,74,1826-1832. 